\title{
Vertical Dynamic Response of Pile Embedded in Layered Transversely Isotropic Soil
}

\author{
Wenbing Wu, ${ }^{1}$ Guosheng Jiang, ${ }^{1}$ Shenggen Huang, ${ }^{1}$ and Chin Jian Leo ${ }^{2}$ \\ ${ }^{1}$ Engineering Faculty, China University of Geosciences, Wuhan, Hubei 430074, China \\ ${ }^{2}$ School of Computing, Engineering and Mathematics, University of Western Sydney, Locked Bag 1797, Penrith, \\ Sydney, NSW 2751, Australia \\ Correspondence should be addressed to Wenbing Wu; zjuwwb1126@163.com and Shenggen Huang; huangshgr@163.com
}

Received 15 April 2014; Revised 24 June 2014; Accepted 24 June 2014; Published 17 July 2014

Academic Editor: Sarp Adali

Copyright (C) 2014 Wenbing Wu et al. This is an open access article distributed under the Creative Commons Attribution License, which permits unrestricted use, distribution, and reproduction in any medium, provided the original work is properly cited.

\begin{abstract}
The dynamic response of pile embedded in layered transversely isotropic soil and subjected to arbitrary vertical harmonic force is investigated. Based on the viscoelastic constitutive relations for a transversely isotropic medium, the dynamic governing equation of the transversely isotropic soil is obtained in cylindrical coordinates. By introducing the fictitious soil pile model and the distributed Voigt model, the governing equations of soil-pile system are also derived. Firstly, the vertical response of the soil layer is solved by using the Laplace transform technique and the separation of variables technique. Secondly, the analytical solution of velocity response in the frequency domain and its corresponding semianalytical solution of velocity response in the time domain are derived by means of inverse Fourier transform and convolution theorem. Finally, based on the obtained solutions, a parametric study has been conducted to investigate the influence of the soil anisotropy on the vertical dynamic response of pile. It can be seen that the influence of the shear modulus of soil in the vertical plane on the dynamic response of pile is more notable than the influence of the shear modulus of soil in the horizontal plane on the dynamic response of pile.
\end{abstract}

\section{Introduction}

The dynamic interaction of soil-pile system is a complicated contact problem owing to the complexity of soil layer. Therefore, many investigators have paid their attention to develop theoretical models to analyze the soil layer for the dynamic interaction of soil-pile system. Many of these various theoretical models fall into four main categories. (1) The dynamic Winkler model [1-7], which represents the soil as a series of distributed springs attached to the pile, is the first category. The stiffness of the soil "springs" is calculated by assuming that wave energy can only propagate outwards under plane-strain conditions [8]. (2) The plane-strain model [9-16], which assumes that the soil is made up of an infinite number of infinitesimally thin, independent, horizontal, elastic layers that extend to infinity, is the second category. It can be noted that the plane-strain model neglects the strain in the vertical direction and the waves propagate only in the horizontal direction. (3) Various three-dimensional axisymmetric continuum models [17-22], in which the soil layer is three-dimensional and the wave effect of soil is considered, is the third category. Due to the complexity of the three-dimensional axisymmetric continuum models, it is very difficult to promote the use of these models in layered soil. (4) The radially inhomogeneous model [23-26], in which the soil region is assumed as a linear viscoelastic medium composed of two concentric regions, an inner annular region of disturbed medium, and an outer semi-infinite undisturbed region, is the fourth category. It can be seen that the model does not simulate the interaction of soil zones rigorously and it is only an approximate simplified model that may lead to the argument whether the results adequately reflect the real interaction of the soil zones [27].

Most of the previous investigations on the dynamic interaction of soil-pile system had treated soil as an isotropic medium. However, in practice soil deposits possess a certain degree of anisotropy owing to their deposition history resulting in properties that are different in the horizontal 
and vertical direction. The vertical and horizontal differences can be simulated by virtue of a transversely isotropic soil model [28] which a simple isotropic model cannot allow for. Tsai [29] studied the torsional dynamic response of a circular disk on a transversely isotropic half-space by utilizing integral transform technique. It is worth noting that the anisotropic material constants have obvious influence on the resonant amplitude and frequency of vibration. Liu and Novak [30] investigated the dynamic response of single pile embedded in transversely isotropic layered media by means of FEM combining with dynamic stiffness matrices of the soil derived from Green's functions for ring loads. Chen et al. [31] proposed a model of transversely isotropic layered elastic media to study the dynamic horizontal impedances of double piles embedded in the ground and subjected to harmonic sway-rocking loadings at the head of the two piles. Chen et al. [32] studied the transient torsional dynamic response of a pile embedded in transversely isotropic saturated soil by utilizing the Laplace transform. Wang et al. [33] investigated the dynamic response of an end bearing pile embedded in transversely isotropic saturated soil when there was a time-harmonic torsional loading acting on the pile head. Notwithstanding the work above, it can be seen that no investigation corresponding to the vertical dynamic response of a pile embedded in layered transversely isotropic elastic soil has been reported in existing literatures until now.

Based on the above literature review, the main objective of this paper is to develop an extended soil-pile interaction model to simulate the vertical dynamic response of pile embedded in layered transversely isotropic soil. Utilizing the fictitious soil pile model to simulate the dynamic interaction between the pile and pile end soil [34], the governing equations of soil-pile system are established when the vertical wave effect of surrounding soil is taken into account. Then, the analytical solution of velocity response in the frequency domain and its corresponding semianalytical solution of velocity response in the time domain are derived by means of integral transform technique and separation of variables technique. Based on these solutions, a parametric study has been conducted to assess the influence of the soil anisotropy on the vibration behavior of the pile.

\section{Governing Equations}

2.1. Computational Model and Assumptions. The problem studied in this paper is the vertical vibration of viscoelastic pile embedded in layered transversely isotropic soil and the geometric model is shown in Figure 1. Based on the fictitious soil pile model, the soil-pile system is discretized into a total of $m$ segments along the vertical direction, which are numbered by $1,2, \ldots, j, \ldots, m$. The thickness of the $j$ th $(1 \leq$ $j \leq m$ ) soil-pile segment is denoted by $l_{j}$ and the depth of the $j$ th $(1 \leq j \leq m)$ soil layer top is denoted by $h_{j}$. If the numbers of the soil-pile segments are enough, the properties of soil and pile within each segment can be assumed to be homogeneous, respectively. The pile length is denoted by $H^{p}$ and the thickness of pile end soil is denoted by $H^{s} . q(t)$ is an arbitrary vertical harmonic force acting on the pile head.

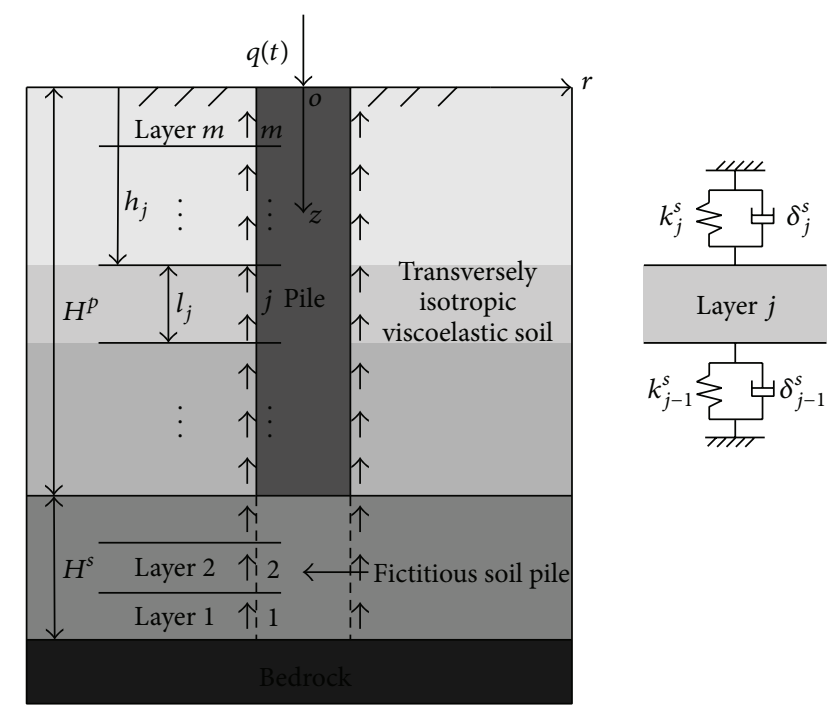

FIGURE 1: Schematic of pile-soil interaction model.

The following assumptions are adopted during the analysis.

(1) The surrounding soil of pile is layered, transversely isotropic and viscoelastic. The damping force of soil is proportional to the strain rate, and the proportional coefficient of the $j$ th soil layer is denoted by $\eta_{j}^{s}$.

(2) The top surface of soil layer is assumed to be free boundary without normal and shear stresses, and the bottom surface of pile end soil is assumed to be rigid boundary without displacements.

(3) The dynamic interaction of the adjacent soil layers is simulated by using the distributed Voigt model. The spring constant and damping coefficient of the distributed Voigt model between the $j$ th soil layer and its upper adjacent soil layer are denoted by $k_{j}^{s}$ and $\delta_{j}^{s}$, and the corresponding values between the $j$ th soil layer and its lower adjacent soil layer are denoted by $k_{j-1}^{s}$ and $\delta_{j-1}^{s}$, respectively.

(4) During vibration, the vertical wave effect of surrounding soil is taken into account, but the radial wave effect of surrounding soil is ignored.

(5) Both the pile and the fictitious soil pile are vertical, viscoelastic, and circular in cross-section and have a perfect contact with the surrounding soil during vibration.

(6) During vibration, the soil-pile system is subjected to small deformations and strains, and the conditions of displacement continuity and force equilibrium are satisfied at the interface of the adjacent pile (including fictitious soil pile) segments.

\subsection{Governing Equations of Soil-Pile System}

2.2.1. Dynamic Equation of Soil. Combining with the viscoelastic constitutive relations for a transversely isotropic 
medium proposed by Ding [35], the dynamic equilibrium equation of the transversely isotropic soil in cylindrical coordinates can be derived as follows

$$
\begin{aligned}
& {\left[C_{11}\left(\frac{\partial^{2}}{\partial r^{2}}+\frac{1}{r} \frac{\partial}{\partial r}-\frac{1}{r^{2}}\right)+C_{66} \frac{1}{r^{2}} \frac{\partial^{2}}{\partial \theta^{2}}+C_{44} \frac{\partial^{2}}{\partial z^{2}}\right] u_{r}} \\
& +\left[\frac{C_{11}-C_{66}}{r} \frac{\partial^{2}}{\partial r \partial \theta}-\frac{C_{11}+C_{66}}{r^{2}} \frac{\partial}{\partial \theta}\right] u_{\theta} \\
& +\left(C_{13}+C_{44}\right) \frac{\partial^{2} w}{\partial r \partial z}=\rho^{s} \frac{\partial^{2} u_{r}}{\partial t^{2}}, \\
& {\left[\left(C_{11}-C_{66}\right) \frac{1}{r} \frac{\partial^{2}}{\partial r \partial \theta}+\left(C_{11}+C_{66}\right) \frac{1}{r^{2}} \frac{\partial}{\partial \theta}\right] u_{r}} \\
& +\left(C_{13}+C_{44}\right) \frac{1}{r} \frac{\partial^{2} w}{\partial \theta \partial z} \\
& +\left[C_{66}\left(\frac{\partial^{2}}{\partial r^{2}}+\frac{1}{r} \frac{\partial}{\partial r}-\frac{1}{r^{2}}\right)+C_{11} \frac{1}{r^{2}} \frac{\partial^{2}}{\partial \theta^{2}}+C_{44} \frac{\partial^{2}}{\partial z^{2}}\right] u_{\theta} \\
& =\rho^{s} \frac{\partial^{2} u_{\theta}}{\partial t^{2}}, \\
& \left(C_{13}+C_{44}\right)\left(\frac{\partial^{2}}{\partial r \partial z}+\frac{1}{r} \frac{\partial}{\partial z}\right) u_{r}+\left(C_{13}+C_{44}\right) \frac{1}{r} \frac{\partial^{2} u_{\theta}}{\partial \theta \partial z} \\
& +\left[C_{44}\left(\frac{\partial^{2}}{\partial r^{2}}+\frac{1}{r} \frac{\partial}{\partial r}+\frac{1}{r^{2}} \frac{\partial^{2}}{\partial \theta^{2}}\right)+C_{33} \frac{\partial^{2}}{\partial z^{2}}\right] w \\
& =\rho^{s} \frac{\partial^{2} w}{\partial t^{2}},
\end{aligned}
$$

where $u_{r}, u_{\theta}$, and $w$ denote the radial displacement, circumferential displacement, and vertical displacement, respectively. The coefficients of the above equations should satisfy the following equations

$$
\begin{aligned}
& C_{11}=C_{22}=\frac{E_{h}^{s}\left(1-\mu_{h \nu}^{s} \mu_{v h}^{s}\right)}{\left(1+\mu_{h h}^{s}\right)\left(1-\mu_{h h}^{s}-2 \mu_{h v}^{s} \mu_{v h}^{s}\right)}, \\
& C_{12}=\frac{E_{h}^{s}\left(\mu_{h h}^{s}+\mu_{h \nu}^{s} \mu_{v h}^{s}\right)}{\left(1+\mu_{h h}^{s}\right)\left(1-\mu_{h h}^{s}-2 \mu_{h v}^{s} \mu_{v h}^{s}\right)}, \\
& C_{13}=C_{23}=\frac{E_{h}^{s} \mu_{h h}^{s}}{1-\mu_{h h}^{s}-2 \mu_{h v}^{s} \mu_{v h}^{s}}, \\
& C_{33}=\frac{E_{v}\left(1-\mu_{h h}^{s}\right)}{1-\mu_{h h}^{s}-2 \mu_{h v}^{s} \mu_{v h}^{s}}, \\
& C_{44}=C_{55}=G_{v}^{s}, \\
& C_{66}=\frac{\left(C_{11}-C_{22}\right)}{2},
\end{aligned}
$$

where $E_{h}^{s}$ and $E_{v}^{s}$ denote the horizontal and vertical elastic modulus, respectively. $G_{v}^{s}$ denotes the shear modulus in the vertical plane. $\mu_{h v}^{s}$ is the Poisson's ration in the vertical direction caused by the horizontal stress and $\mu_{v h}^{s}$ is the Poisson's ratio in the horizontal direction caused by the vertical stress and $\mu_{h v}^{s}$ and $\mu_{v h}^{s}$ should satisfy $\mu_{v h}^{s} / E_{v}^{s}=\mu_{h v}^{s} / E_{h}^{s}$. $\mu_{h h}^{s}$ is the Poisson's ratio in the orthogonal direction of the horizontal strain caused by the horizontal stress.

Owing to the assumption that only the vertical wave effect of surrounding soil is taken into consideration, the equilibrium equation of the transversely isotropic soil for the axisymmetric problem can be further rewritten as follows

$$
C_{33} \frac{\partial^{2} w}{\partial z^{2}}+C_{44}\left[\frac{\partial^{2} w}{\partial r^{2}}+\frac{1}{r} \frac{\partial w}{\partial r}\right]=\rho^{s} \frac{\partial^{2} w}{\partial t^{2}} .
$$

Based on (3) and taking into account the viscosity of soil, the governing equation of the $j$ th transversely isotropic soil layer for the axisymmetric problem can be established as

$$
\begin{gathered}
\delta_{j}^{s} \frac{\partial^{2} w_{j}}{\partial z^{2}}+\frac{\eta_{j}^{s}}{G_{v j}^{s}} \frac{\partial}{\partial t}\left(\frac{\partial^{2} w_{j}}{\partial z^{2}}\right)+\left(\frac{1}{r} \frac{\partial w_{j}}{\partial r}+\frac{\partial^{2} w_{j}}{\partial r^{2}}\right) \\
+\frac{\eta_{j}^{s}}{G_{v j}^{s}} \frac{\partial}{\partial t}\left(\frac{1}{r} \frac{\partial w_{j}}{\partial r}+\frac{\partial^{2} w_{j}}{\partial r^{2}}\right)=\frac{\rho_{j}^{s}}{G_{v j}^{s}} \frac{\partial^{2} w_{j}}{\partial t^{2}},
\end{gathered}
$$

where $w_{j}=w_{j}(r, z, t)$ is the vertical displacement of the $j$ th soil layer. $\eta_{j}^{s}, \rho_{j}^{s}$, and $G_{v j}^{s}$ denote the viscous damping coefficient, density, and shear modulus in the vertical plane of the $j$ th soil layer. $\delta_{j}^{s}=2\left(1-\mu_{h h j}^{s}\right)\left(1+\mu_{v h j}^{s}\right) /\left(1-\mu_{h h j}^{s}-\right.$ $\left.2\left(\mu_{v h j}^{s}\right)^{2} \kappa_{j}^{s}\right) \cdot \kappa_{j}^{s}=\left(1+\mu_{h h j}^{s}\right) G_{h j}^{s} /\left(1+\mu_{v h j}^{s}\right) G_{v j}^{s}$ denotes the ratio of the elastic modulus of the $j$ th soil layer in the horizontal direction to that in the vertical direction; $G_{h j}^{s}$, $\mu_{v h j}^{s}$, and $\mu_{h h j}^{s}$ denote the shear modulus in the horizontal plane, the Poisson's ratio in the horizontal direction caused by the vertical stress, and the Poisson's ratio in the orthogonal direction of the horizontal strain caused by the horizontal stress of the $j$ th soil layer, respectively.

2.2.2. Dynamic Equation of Pile. Denoting $u_{j}=u_{j}(z, t)$ to be the vertical displacement of the $j$ th pile (including fictitious soil pile) segment and according to the Euler-Bernoulli rod theory, the dynamic equilibrium equation of pile can be established as

$$
\begin{gathered}
E_{j}^{p} A_{j}^{p} \frac{\partial^{2} u_{j}}{\partial z^{2}}+A_{j}^{p} \eta_{j}^{p} \frac{\partial^{3} u_{j}}{\partial t \partial z^{2}}-m_{j}^{p} \frac{\partial^{2} u_{j}}{\partial t^{2}} \\
-2 \pi r_{j}^{p} \tau_{r z j}^{s}\left(r_{j}^{p}, z, t\right)=0,
\end{gathered}
$$

where $E_{j}^{p}=\rho_{j}^{p}\left(V_{j}^{p}\right)^{2}, A_{j}^{p}=\pi\left(r_{j}^{p}\right)^{2}, r_{j}^{p}, m_{j}^{p}, \rho_{j}^{p}, V_{j}^{p}$, and $\eta_{j}^{p}$ denote the elastic modulus, cross-section area, radius, mass per unit length of pile (including fictitious soil pile), density, elastic longitudinal wave velocity, and viscous damping coefficient of the $j$ th pile (including fictitious soil pile) segment, respectively. $\tau_{r z j}^{s}\left(r_{j}^{p}, z, t\right)$ is the frictional force of the $j$ th soil layer acting on the surface of the $j$ th pile shaft and can be expressed as

$$
\tau_{r z j}^{s}\left(r_{j}^{p}, z, t\right)=G_{v j}^{s} \frac{\partial w_{j}\left(r_{j}^{p}, z, t\right)}{\partial r}+\eta_{j}^{s} \frac{\partial^{2} w_{j}\left(r_{j}^{p}, z, t\right)}{\partial t \partial r} .
$$


Combining with the assumptions, the boundary and initial conditions of soil-pile system can be established as follows.

(1) Boundary Conditions of Soil. At the top surface of the $j$ th soil layer,

$$
\left.E_{v j}^{s} \frac{\partial w_{j}}{\partial z}\right|_{z=h_{j}}=\left.\left(k_{j}^{s} w_{j}+\delta_{j}^{s} \frac{\partial w_{j}}{\partial t}\right)\right|_{z=h_{j}}
$$

At the bottom surface of the $j$ th soil layer,

$$
\left.E_{v j}^{s} \frac{\partial w_{j}}{\partial z}\right|_{z=h_{j}+l_{j}}=-\left.\left(k_{j-1}^{s} w_{j}+\delta_{j-1}^{s} \frac{\partial w_{j}}{\partial t}\right)\right|_{z=h_{j}+l_{j}} .
$$

At an infinite radial distance of the $j$ th soil layer,

$$
\sigma_{j}(\infty, z)=0, \quad w_{j}(\infty, z)=0 .
$$

(2) Boundary Conditions of Pile. At the top surface of the $j$ th pile (including fictitious soil pile) segment,

$$
\left.\frac{\partial u_{j}}{\partial z}\right|_{z=h_{j}}=-\left.\frac{Z_{j}(s) u_{j}}{E_{j}^{p} A_{j}^{p}}\right|_{z=h_{j}} .
$$

At the bottom surface of the $j$ th pile (including fictitious soil pile) segment,

$$
\left.\frac{\partial u_{j}}{\partial z}\right|_{z=h_{j}+l_{j}}=-\left.\frac{Z_{j-1}(s) u_{j}}{E_{j}^{p} A_{j}^{p}}\right|_{z=h_{j}+l_{j}},
$$

where $Z_{j}(s)$ and $Z_{j-1}(s)$ denote the displacement impedance function at the top and bottom surfaces of the $j$ th pile (including fictitious soil pile) segment. $s$ is the Laplace transform parameter.

(3) Boundary C at the Interface of Soil-Pile System

$$
w\left(r_{j}^{p}, z, t\right)=u_{j}(z, t)
$$

(4) Initial Conditions of Soil-Pile System. Initial conditions of the $j$ th soil layer are as follows:

$$
\left.w_{j}\right|_{t=0}=0,\left.\quad \frac{\partial w_{j}}{\partial t}\right|_{t=0}=0 .
$$

Initial conditions of the $j$ th pile (including fictitious soil pile) segment are as follows:

$$
\left.u_{j}\right|_{t=0}=0,\left.\quad \frac{\partial u_{j}}{\partial t}\right|_{t=0}=0 .
$$

\section{Solutions of the Governing Equations}

3.1. Vibrations of the Soil Layer. Denoting $W_{j}(r, z, s)=$ $\int_{0}^{+\infty} w_{j}(r, z, t) e^{-s t} \mathrm{~d} t$ to be the Laplace transform with respect to time of $w_{j}(r, z, t)$ and associating with the initial condition (10a), (4) is transformed by using the Laplace transform technique as follows

$$
\begin{aligned}
& \left(\delta_{j}^{s}+\frac{\eta_{j}^{s} \cdot s}{G_{v j}^{s}}\right) \frac{\partial^{2} W_{j}}{\partial z^{2}}+\left(1+\frac{\eta_{j}^{s} \cdot s}{G_{v j}^{s}}\right)\left(\frac{1}{r} \frac{\partial W_{j}}{\partial r}+\frac{\partial^{2} W_{j}}{\partial r^{2}}\right) \\
& =\left(\frac{s}{V_{v j}^{s}}\right)^{2} W_{j},
\end{aligned}
$$

where $V_{v j}^{s}=\sqrt{G_{v j}^{s} / \rho_{j}^{s}}$ is the shear wave velocity of the $j$ th soil layer in the vertical direction.

By virtue of the separation of variables technique and denoting $W_{j}(r, z, s)=R_{j}(r, s) Z_{j}(z, s)$, (11) can be decoupled as follows

$$
\begin{gathered}
\frac{\mathrm{d}^{2} R_{j}(r, s)}{\mathrm{d} r^{2}}+\frac{1}{r} \frac{\mathrm{d} R_{j}(r, s)}{\mathrm{d} r}-\xi_{j}^{2} R_{j}(r, s)=0, \\
\frac{\mathrm{d}^{2} Z_{j}(z, s)}{\mathrm{d} z^{2}}+\beta_{j}^{2} Z_{j}(z, s)=0,
\end{gathered}
$$

where constants $\xi_{j}$ and $\beta_{j}$ must satisfy the following equation

$$
\xi_{j}^{2}=\frac{\left(\delta_{j}^{s}+\eta_{j}^{s} \cdot s / G_{v j}^{s}\right) \beta_{j}^{2}+\left(s / V_{v j}^{s}\right)^{2}}{\left(1+\eta_{j}^{s} \cdot s / G_{v j}^{s}\right)} .
$$

It can be seen that (12) is Bessel equation and (13) is ordinary differential equation of second order whose general solutions can be easily obtained. Associating with these general solutions, the vertical displacement of the $j$ th soil layer, $W_{j}(r, z, s)$ can be derived as

$$
\begin{aligned}
W_{j}(r, z, s)=[ & \left.A_{j} K_{0}\left(\xi_{j} r\right)+B_{j} I_{0}\left(\xi_{j} r\right)\right] \\
& \times\left[C_{j} \sin \left(\beta_{j} z\right)+D_{j} \cos \left(\beta_{j} z\right)\right],
\end{aligned}
$$

where $I_{0}(\cdot)$ and $K_{0}(\cdot)$ denote the modified Bessel functions of order zero of the first and second kind, respectively. $A_{j}$, $B_{j}, C_{j}$, and $D_{j}$ are constants determined by the boundary conditions.

Converting $z=h_{j}$ and $z=h_{j}+l_{j}$ in the global coordinates into $z^{\prime}=0$ and $z^{\prime}=l_{j}$ in the local coordinates, $(7 \mathrm{a})$, 
(7b), and (7c) are transformed by using the Laplace transform technique and can be rewritten as follows

$$
\begin{gathered}
{\left.\left[\frac{\left(k_{j}^{s}+\delta_{j}^{s} \cdot s\right)}{E_{v j}^{s}} W_{j}\left(r, z^{\prime}, s\right)-\frac{\partial W_{j}\left(r, z^{\prime}, s\right)}{\partial z^{\prime}}\right]\right|_{z^{\prime}=0}=0, \quad(16 \mathrm{a})} \\
{\left.\left[\frac{\left(k_{j-1}^{s}+\delta_{j-1}^{s} \cdot s\right)}{E_{v j}^{s}} W_{j}\left(r, z^{\prime}, s\right)+\frac{\partial W_{j}\left(r, z^{\prime}, s\right)}{\partial z^{\prime}}\right]\right|_{z^{\prime}=l_{j}}=0,} \\
\bar{\sigma}_{j}\left(\infty, z^{\prime}\right)=0, \quad W_{j}\left(\infty, z^{\prime}\right)=0 . \quad(16 \mathrm{~b})
\end{gathered}
$$

According to the properties of the modified Bessel functions, that is, if $r \rightarrow \infty$, then $I_{n}(\cdot) \rightarrow \infty$ and $K_{n}(\cdot) \rightarrow 0$, it can be obtained that $B_{j}=0$ from boundary conditions (16c). By means of boundary conditions (16a) and (16b), the following equation can be obtained

$$
\begin{aligned}
\tan & \left(\beta_{j} l_{j}\right) \\
= & \left(\frac{k_{j}^{s}+\delta_{j}^{s} \cdot s}{E_{v j}^{s}} l_{j}+\frac{k_{j-1}^{s}+\delta_{j-1}^{s} \cdot s}{E_{v j}^{s}} l_{j}\right) \beta_{j} l_{j} \\
& \times\left(\left(\beta_{j} l_{j}\right)^{2}-\left(\frac{k_{j}^{s}+\delta_{j}^{s} \cdot s}{E_{v j}^{s}} l_{j}\right)\left(\frac{k_{j-1}^{s}+\delta_{j-1}^{s} \cdot s}{E_{v j}^{s}} l_{j}\right)\right)^{-1} \\
= & \frac{\left(\bar{K}_{j}+\bar{K}_{j}^{\prime}\right) \beta_{j} l_{j}}{\left(\beta_{j} l_{j}\right)^{2}-\bar{K}_{j} \bar{K}_{j}^{\prime}}
\end{aligned}
$$

where $\bar{K}_{j}=\left(\left(k_{j}^{s}+\delta_{j}^{s} \cdot s\right) / E_{v j}^{s}\right) l_{j}$ and $\bar{K}_{j}^{\prime}=\left(\left(k_{j-1}^{s}+\right.\right.$ $\left.\left.\delta_{j-1}^{s} \cdot s\right) / E_{v j}^{s}\right) l_{j}$ denote the dimensionless complex stiffness of the upper surface and lower surface of the $j$ th soil layer. Substituting $s=i \omega$ into (17) and solving it by using bisection method in the frequency domain, a series of eigenvalues, $\beta_{j n}$, can be derived. $\xi_{j n}$ can also be derived by substituting $\beta_{j n}$ into (14).

Then, the vertical displacement of the $j$ th soil layer can be rewritten as

$$
W_{j}\left(r, z^{\prime}, s\right)=\sum_{n=1}^{\infty} A_{j n} K_{0}\left(\xi_{j n} r\right) \sin \left(\beta_{j n} z^{\prime}+\phi_{j n}\right),
$$

where $\phi_{j n}=\arctan \left(\beta_{j n} l_{j} / \bar{K}_{j}\right)$ and $A_{j n}$ are a series of constants determined by the boundary conditions which reflect the dynamic interaction of soil and pile.

3.2. Vibrations of the Pile. Denoting $U_{j}(z, s)$ to be the Laplace transform with respect to time of $u_{j}(z, t),(5)$ is transformed by using the Laplace transform technique and can be rewritten combining with (6) and (18)

$$
\begin{aligned}
&\left(V_{j}^{p}\right)^{2}\left(1+\frac{\eta_{j}^{p}}{E_{j}^{p}} \cdot s\right) \frac{\partial^{2} U_{j}}{\partial z^{\prime 2}}-s^{2} U_{j} \\
&-\frac{2 \pi r_{j}^{p}}{\rho_{j}^{p} A_{j}^{p}}\left(G_{v j}^{s}+\eta_{v j}^{s} \cdot s\right) \sum_{n=1}^{\infty} A_{j n} \xi_{j n} K_{1}\left(\xi_{j n} r_{j}^{p}\right) \\
& \times \sin \left(\beta_{j n} z^{\prime}+\phi_{j n}\right)=0 .
\end{aligned}
$$

It is not difficult to obtain that the general solution of (19) can be expressed as

$$
\begin{aligned}
U_{j}= & M_{j}\left[\cos \left(\frac{\bar{\lambda}_{j} z^{\prime}}{l_{j}}\right)+\sum_{n=1}^{\infty} \chi_{j n}^{\prime} \sin \left(\beta_{j n} z^{\prime}+\phi_{j n}\right)\right] \\
& +N_{j}\left[\sin \left(\frac{\bar{\lambda}_{j} z^{\prime}}{l_{j}}\right)+\sum_{n=1}^{\infty} \chi_{j n}^{\prime \prime} \sin \left(\beta_{j n} z^{\prime}+\phi_{j n}\right)\right],
\end{aligned}
$$

where

$$
\begin{aligned}
& \chi_{j n}^{\prime}=\chi_{j n}\left[\frac{\cos \left(\bar{\beta}_{j n}+\bar{\lambda}_{j}+\phi_{j n}\right)-\cos \phi_{j n}}{\bar{\beta}_{j n}+\bar{\lambda}_{j}}\right. \\
& \left.+\frac{\cos \left(\bar{\beta}_{j n}-\bar{\lambda}_{j}+\phi_{j n}\right)-\cos \phi_{j n}}{\bar{\beta}_{j n}-\bar{\lambda}_{j}}\right], \\
& \chi_{j n}^{\prime \prime}=\chi_{j n}\left[\frac{\sin \left(\bar{\beta}_{j n}+\bar{\lambda}_{j}+\phi_{j n}\right)-\sin \phi_{j n}}{\bar{\beta}_{j n}+\bar{\lambda}_{j}}\right. \\
& \left.-\frac{\sin \left(\bar{\beta}_{j n}-\bar{\lambda}_{j}+\phi_{j n}\right)-\sin \phi_{j n}}{\bar{\beta}_{j n}-\bar{\lambda}_{j}}\right] \\
& \chi_{j n}=\frac{\left(G_{v j}^{s}+\eta_{j}^{s} \cdot s\right) \bar{\xi}_{j n} K_{1}\left(\bar{\xi}_{j n} \bar{r}_{j}^{p}\right) t_{j}^{2}}{\rho_{j}^{p} l_{j} \bar{r}_{j}^{p}\left[\bar{\beta}_{j n}^{2}\left(1+\left(\eta_{j}^{p} / E_{j}^{p}\right) \cdot s\right)+s^{2} t_{j}^{2}\right] \varphi_{j n} L_{j n}}, \\
& \varphi_{j n}=K_{0}\left(\bar{\xi}_{j n} \bar{r}_{j}^{p}\right)+\frac{2\left(G_{v j}^{s}+\eta_{j}^{s} \cdot s\right) \bar{\xi}_{j n} K_{1}\left(\bar{\xi}_{j n} \bar{r}_{j}^{p}\right) t_{j}^{2}}{\rho_{j}^{p} l_{j}^{2} \bar{r}_{j}^{p}\left[\bar{\beta}_{j n}^{2}\left(1+\left(\eta_{j}^{p} / E_{j}^{p}\right) \cdot s\right)+s^{2} t_{j}^{2}\right]}, \\
& L_{j n}=\int_{0}^{l_{j}} \sin ^{2}\left(\beta_{j n} z^{\prime}+\phi_{j n}\right) \mathrm{d} z^{\prime},
\end{aligned}
$$

where $\bar{\lambda}_{j}=\sqrt{-s^{2} t_{j}^{2} /\left(1+\left(\eta_{j}^{p} / E_{j}^{p}\right) \cdot s\right)}, \bar{\beta}_{j n}=\beta_{j n} l_{j}, \bar{\xi}_{j n}=\xi_{j n} l_{j}$, and $\bar{r}_{j}^{p}=r_{j}^{p} / l_{j}$ are all dimensionless parameters. $t_{j}=l_{j} / V_{j}^{p}$ denotes the propagation time of elastic longitudinal wave in the $j$ th pile segment. $K_{1}(\cdot)$ is the modified Bessel functions of order one of the second kind. 
Combining with the boundary conditions (8a) and (8b), the displacement impedance function at the head of the $j$ th pile segment can be derived in the local coordinates as follows

$$
\begin{aligned}
Z_{j}(s)= & \frac{-\left.E_{j}^{p} A_{j}^{p}\left(\partial U_{j} / \partial z^{\prime}\right)\right|_{z^{\prime}=0}}{\left.U_{j}\right|_{z^{\prime}=0}}=-\frac{E_{j}^{p} A_{j}^{p}}{l_{j}} \\
& \times\left(\frac{M_{j}}{N_{j}} \sum_{n=1}^{\infty} \chi_{j n}^{\prime} \bar{\beta}_{j n} \cos \phi_{j n}\right. \\
& \left.+\bar{\lambda}_{j}+\sum_{n=1}^{\infty} \chi_{j n}^{\prime \prime} \bar{\beta}_{j n} \cos \phi_{j n}\right) \\
& \times\left(\frac{M_{j}}{N_{j}}\left(1+\sum_{n=1}^{\infty} \chi_{j n}^{\prime} \sin \phi_{j n}\right)\right. \\
& \left.+\sum_{n=1}^{\infty} \chi_{j n}^{\prime \prime} \sin \phi_{j n}\right)^{-1}
\end{aligned}
$$

where

$$
\begin{aligned}
\frac{M_{j}}{N_{j}}=\left(\sum_{n=1}^{\infty} \chi_{j n}^{\prime \prime} \bar{\beta}_{j n} \cos \left(\bar{\beta}_{j n}+\phi_{j n}\right)+\bar{\lambda}_{j} \cos \bar{\lambda}_{j}\right. \\
\left.\quad+\frac{Z_{j-1}(s) l_{j}}{E_{j}^{p} A_{j}^{p}}\left[\sin \bar{\lambda}_{j}+\sum_{n=1}^{\infty} \chi_{j n}^{\prime \prime} \sin \left(\bar{\beta}_{j n}+\phi_{j n}\right)\right]\right) \\
\quad \times\left(\sum_{n=1}^{\infty} \chi_{j n}^{\prime} \bar{\beta}_{j n} \cos \left(\bar{\beta}_{j n}+\phi_{j n}\right)-\bar{\lambda}_{j} \sin \bar{\lambda}_{j}\right. \\
\left.\quad+\frac{Z_{j-1}(s) l_{j}}{E_{j}^{p} A_{j}^{p}}\left[\cos \bar{\lambda}_{j}+\sum_{n=1}^{\infty} \chi_{j n}^{\prime} \sin \left(\bar{\beta}_{j n}+\phi_{j n}\right)\right]\right)^{-1},
\end{aligned}
$$

where $Z_{j-1}(s)$ denotes the displacement impedance function at the head of the $(j-1)$ th pile segment which can be obtained by using boundary conditions. Then, following the method of recursion typically used in the transfer function technique, the displacement impedance function at the head of pile can be derived as

$$
\begin{aligned}
Z_{m}(s)= & \frac{-\left.E_{m}^{p} A_{m}^{p}\left(\partial U_{m} / \partial z^{\prime}\right)\right|_{z^{\prime}=0}}{\left.U_{m}\right|_{z^{\prime}=0}} \\
= & -\frac{E_{m}^{p} A_{m}^{p}}{l_{m}} \\
& \times\left(\frac{M_{m}}{N_{m}} \sum_{n=1}^{\infty} \chi_{m n}^{\prime} \bar{\beta}_{m n} \cos \phi_{m n}\right. \\
& \left.+\bar{\lambda}_{m}+\sum_{n=1}^{\infty} \chi_{m n}^{\prime \prime} \bar{\beta}_{m n} \cos \phi_{m n}\right)
\end{aligned}
$$

$$
\begin{gathered}
\times\left(\frac{M_{m}}{N_{m}}\left(1+\sum_{n=1}^{\infty} \chi_{m n}^{\prime} \sin \phi_{m n}\right)\right. \\
\left.+\sum_{n=1}^{\infty} \chi_{m n}^{\prime \prime} \sin \phi_{m n}\right)^{-1},
\end{gathered}
$$

where

$$
\begin{aligned}
& \frac{M_{m}}{N_{m}} \\
& =-\left(\sum_{n=1}^{\infty} \chi_{m n}^{\prime \prime} \bar{\beta}_{m n} \cos \left(\bar{\beta}_{m n}+\phi_{m n}\right)+\bar{\lambda}_{m} \cos \bar{\lambda}_{m}\right. \\
& \left.+\frac{Z_{m-1}(s) l_{m}}{E_{m}^{p} A_{m}^{p}}\left[\sin \bar{\lambda}_{m}+\sum_{n=1}^{\infty} \chi_{m n}^{\prime \prime} \sin \left(\bar{\beta}_{m n}+\phi_{m n}\right)\right]\right) \\
& \times\left(\sum_{n=1}^{\infty} \chi_{m n}^{\prime} \bar{\beta}_{m n} \cos \left(\bar{\beta}_{m n}+\phi_{m n}\right)-\bar{\lambda}_{m} \sin \bar{\lambda}_{m}\right. \\
& \left.+\frac{Z_{m-1}(s) l_{m}}{E_{m}^{p} A_{m}^{p}}\left[\cos \bar{\lambda}_{m}+\sum_{n=1}^{\infty} \chi_{m n}^{\prime} \sin \left(\bar{\beta}_{m n}+\phi_{m n}\right)\right]\right)^{-1}, \\
& \chi_{m n}^{\prime}=\chi_{m n}\left[\frac{\cos \left(\bar{\beta}_{m n}+\bar{\lambda}_{m}+\phi_{m n}\right)-\cos \phi_{m n}}{\bar{\beta}_{m n}+\bar{\lambda}_{m}}\right. \\
& \left.+\frac{\cos \left(\bar{\beta}_{m n}-\bar{\lambda}_{m}+\phi_{m n}\right)-\cos \phi_{m n}}{\bar{\beta}_{m n}-\bar{\lambda}_{m}}\right], \\
& \chi_{m n}^{\prime \prime}=\chi_{m n}\left[\frac{\sin \left(\bar{\beta}_{m n}+\bar{\lambda}_{m}+\phi_{m n}\right)-\sin \phi_{m n}}{\bar{\beta}_{m n}+\bar{\lambda}_{m}}\right. \\
& \left.-\frac{\sin \left(\bar{\beta}_{m n}-\bar{\lambda}_{m}+\phi_{m n}\right)-\sin \phi_{m n}}{\bar{\beta}_{m n}-\bar{\lambda}_{m}}\right] \\
& \chi_{m n}=\frac{\left(G_{v m}^{s}+\eta_{m}^{s} \cdot s\right) \bar{\xi}_{m n} K_{1}\left(\bar{\xi}_{m n} \bar{r}_{m}^{p}\right) t_{m}^{2}}{\rho_{m}^{p} l_{m} \bar{r}_{m}^{p}\left[\bar{\beta}_{m n}^{2}\left(1+\left(\eta_{m}^{p} / E_{m}^{p}\right) \cdot s\right)+s^{2} t_{m}^{2}\right] \varphi_{m n} L_{m n}}, \\
& \varphi_{m n}=K_{0}\left(\bar{\xi}_{m n} \bar{r}_{m}^{p}\right) \\
& +\frac{2\left(G_{v m}^{s}+\eta_{m}^{s} \cdot s\right) \bar{\xi}_{m n} K_{1}\left(\bar{\xi}_{m n} \bar{r}_{m}^{p}\right) t_{m}^{2}}{\rho_{m}^{p} l_{m}^{2} \bar{r}_{m}^{p}\left[\bar{\beta}_{m n}^{2}\left(1+\left(\eta_{m}^{p} / E_{m}^{p}\right) \cdot s\right)+s^{2} t_{m}^{2}\right]}, \\
& L_{m n}=\int_{0}^{l_{m}} \sin ^{2}\left(\beta_{m n} z^{\prime}+\phi_{m n}\right) \mathrm{d} z^{\prime},
\end{aligned}
$$

where $\bar{\lambda}_{m}=\sqrt{-s^{2} t_{m}^{2} /\left(1+\left(\eta_{m}^{p} / E_{m}^{p}\right) \cdot s\right)}, \bar{\beta}_{m n}=\beta_{m n} l_{m}, \bar{\xi}_{m n}=$ $\xi_{m n} l_{m}$, and $\bar{r}_{m}^{p}=r_{m}^{p} / l_{m}$ are all dimensionless parameters. $t_{m}=$ $l_{m} / V_{m}^{p}$ denotes the propagation time of elastic longitudinal 
wave in the $m$ th pile segment. $\phi_{m n}$ and $\beta_{m n}$ can be obtained from the following equations

$$
\begin{gathered}
\phi_{m n}=\arctan \left(\frac{\beta_{m n} l_{m}}{\bar{K}_{m}}\right), \\
\tan \left(\beta_{m} l_{m}\right)=\frac{\left(\bar{K}_{m}+\bar{K}_{m}^{\prime}\right) \beta_{m} l_{m}}{\left(\beta_{m} l_{m}\right)^{2}-\bar{K}_{m} \bar{K}_{m}^{\prime}}
\end{gathered}
$$

where $\bar{K}_{m}=\left(\left(k_{m}^{s}+\delta_{m}^{s} \cdot s\right) / E_{v m}^{s}\right) l_{m}$ and $\bar{K}_{m}^{\prime}=\left(\left(k_{m-1}^{s}+\delta_{m-1}^{s}\right.\right.$. $\left.s) / E_{v m}^{s}\right) l_{m}$ denote the dimensionless complex stiffness of the upper surface and lower surface of the $m$ th soil layer.

Then, the velocity transfer function at the head of pile can be obtained as

$$
\begin{aligned}
G_{v}(s)= & \frac{s}{Z_{m}(s)} \\
= & -\frac{l_{m} \cdot s}{E_{m}^{p} A_{m}^{p}}\left(\frac{M_{m}}{N_{m}}\left(1+\sum_{n=1}^{\infty} \chi_{m n}^{\prime} \sin \phi_{m n}\right)\right. \\
& \left.+\sum_{n=1}^{\infty} \chi_{m n}^{\prime \prime} \sin \phi_{m n}\right) \\
& \times\left(\frac{M_{m}}{N_{m}} \sum_{n=1}^{\infty} \chi_{m n}^{\prime} \bar{\beta}_{m n} \cos \phi_{m n}\right. \\
& \left.+\bar{\lambda}_{m}+\sum_{n=1}^{\infty} \chi_{m n}^{\prime \prime} \bar{\beta}_{m n} \cos \phi_{m n}\right)^{-1}
\end{aligned}
$$

Letting $s=i \omega$ and substituting it into (27), the velocity response in the frequency domain at the head of pile can be obtained as

$$
H_{v}(i \omega)=\frac{i \omega}{Z_{2}(i \omega)}=-\frac{1}{\rho_{m}^{p} A_{m}^{p} V_{m}^{p}} H_{v}^{\prime},
$$

where $H_{v}^{\prime}$ is the dimensionless velocity admittance at the pile head which can be expressed as

$$
\begin{array}{r}
H_{v}^{\prime}=i \omega t_{m}\left(\frac{M_{m}}{N_{m}}\left(1+\sum_{n=1}^{\infty} \chi_{m n}^{\prime} \sin \phi_{m n}\right)\right. \\
\left.+\sum_{n=1}^{\infty} \chi_{m n}^{\prime \prime} \sin \phi_{m n}\right) \\
\times\left(\frac{M_{m}}{N_{m}} \sum_{n=1}^{\infty} \chi_{m n}^{\prime} \bar{\beta}_{m n} \cos \phi_{m n}\right. \\
\left.+\bar{\lambda}_{m}+\sum_{n=1}^{\infty} \chi_{m n}^{\prime \prime} \bar{\beta}_{m n} \cos \phi_{m n}\right)^{-1} .
\end{array}
$$

By means of the inverse Fourier transform and convolution theorem, the velocity response in the time domain at the head of pile can be expressed as $V(t)=\operatorname{IFT}\left[Q(\omega) H_{v}(i \omega)\right]$, where $Q(\omega)$ denotes the Fourier transform of $q(t)$, which is the vertical excitation acting on the pile head.
In particular, the excitation can be regarded as a half-sine pulse in the nondestructive detection of pile foundation as follows

$$
q(t)= \begin{cases}Q_{\max } \sin \left(\frac{\pi}{T} t\right), & t \in(0, T) \\ 0, & t \geq T\end{cases}
$$

where $T$ and $Q_{\max }$ denote the duration of the impulse or impulse width and the maximum amplitude of the vertical excitation, respectively. Then, the velocity response in the time domain at the head of pile can be expressed as

$$
\begin{aligned}
V(t) & =q(t) * \operatorname{IFT}\left[H_{v}(i \omega)\right] \\
& =\operatorname{IFT}\left[Q(i \omega) \cdot H_{v}(i \omega)\right]=-\frac{Q_{\max }}{\rho_{m}^{p} A_{m}^{p} V_{m}^{p}} V_{v}^{\prime},
\end{aligned}
$$

where $V_{v}^{\prime}$ is the dimensionless velocity response which can be expressed as

$$
\begin{aligned}
V_{v}^{\prime}=\frac{1}{2} \int_{-\infty}^{\infty} i \bar{\omega} \bar{t}_{m} \\
\quad \times\left(\frac{M_{m}}{N_{m}}\left(1+\sum_{n=1}^{\infty} \chi_{m n}^{\prime} \sin \phi_{m n}\right)+\sum_{n=1}^{\infty} \chi_{m n}^{\prime \prime} \sin \phi_{m n}\right) \\
\quad \times\left(\frac{M_{m}}{N_{m}} \sum_{n=1}^{\infty} \chi_{m n}^{\prime} \bar{\beta}_{m n} \cos \phi_{m n}+\bar{\lambda}_{m}\right. \\
\quad+\frac{\left.\sum_{n=1}^{\infty} \chi_{m n}^{\prime \prime} \bar{\beta}_{m n} \cos \phi_{m n}\right)^{-1}}{\pi^{2}-\bar{T}^{2} \bar{\omega}^{2}} \cdot\left(1+e^{-i \bar{\omega} \bar{T}}\right) e^{i \bar{\omega} \bar{t}} \mathrm{~d} \bar{\omega}
\end{aligned}
$$

where $\bar{\omega}=T_{c} \omega$ denotes the dimensionless frequency. $\bar{T}$ denotes the dimensionless pulse width which should satisfy $\bar{T}=T / T_{c} \cdot \bar{t}$ denotes the dimensional time variable which should satisfy $\bar{t}=t / T_{c}$.

\section{Analysis of Vibration Characteristics}

According to the derivation process shown in the previous section, it can be seen that the difference between the shear modulus in the vertical plane and the shear modulus in the horizontal plane reflects the soil anisotropy. Therefore, based on the solutions, the influence of these two kinds of shear modulus of pile surrounding soil and pile end soil on the dynamic response of pile is studied in detail. Unless otherwise specified, the length, radius, density, and longitudinal wave velocity of pile are $15 \mathrm{~m}, 0.5 \mathrm{~m}, 2500 \mathrm{~kg} / \mathrm{m}^{3}$, and $3800 \mathrm{~m} / \mathrm{s}$, respectively. The spring constant of the distributed Voigt model is equal to the elastic modulus of the lower soil layer, and the damping coefficient of the distributed Voigt model is $10000 \mathrm{~N} \cdot \mathrm{m}^{-3} \cdot \mathrm{s}$. 


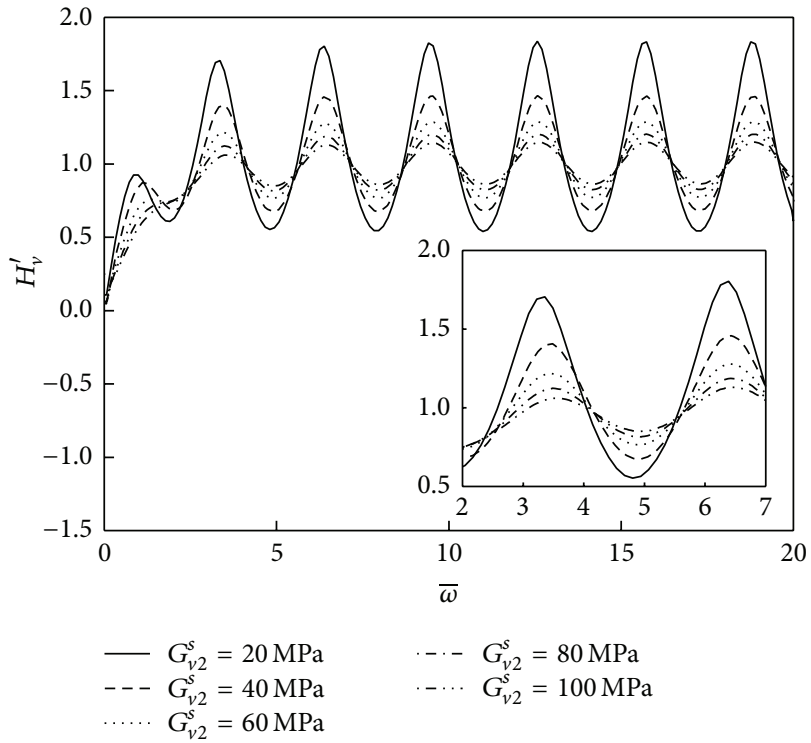

(a) Velocity admittance curves

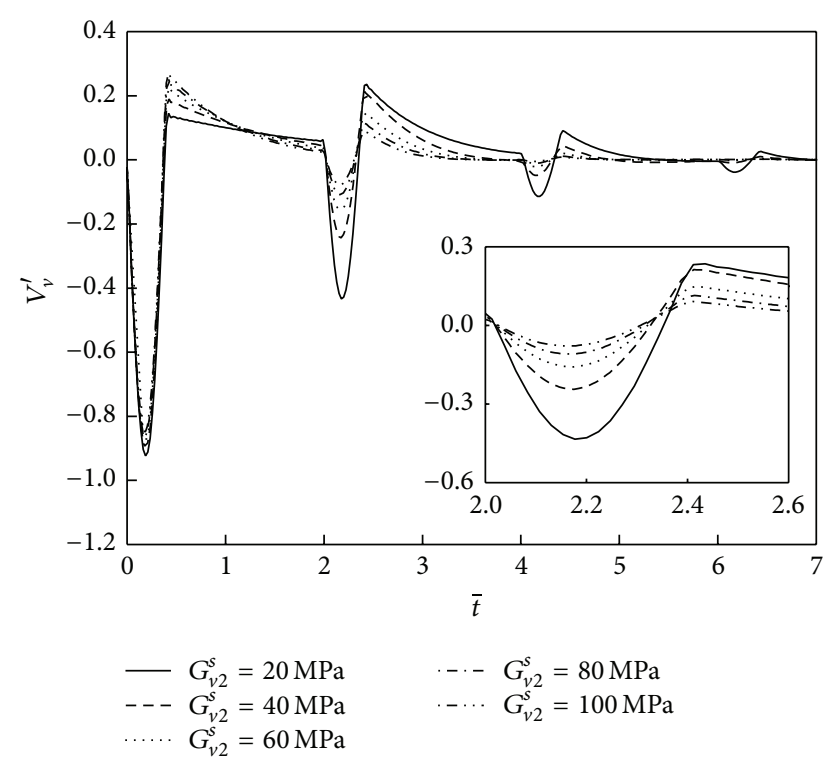

(b) Reflected wave signal curve

FIGURE 2: Influence of the shear modulus of pile surrounding soil in the vertical plane on the dynamic response of pile.

4.1. Influence of the Anisotropy of Pile Surrounding Soil on the Dynamic Response of Pile. Firstly, the influence of the shear modulus of pile surrounding soil in the vertical plane on the dynamic response of pile is investigated. Parameters of pile end soil are as follows: the thickness is three times that of pile diameter; the soil density is $2000 \mathrm{~kg} / \mathrm{m}^{3}$; both the shear modulus in the vertical plane and the shear modulus in the horizontal plane are $120 \mathrm{MPa}$; both the Poisson's ratio in the horizontal direction caused by the vertical stress and the Poisson's ratio in the orthogonal direction of the horizontal strain caused by the horizontal stress are 0.35 ; the damping coefficient is $1000 \mathrm{~N} \cdot \mathrm{m}^{-3} \cdot \mathrm{s}$. Parameters of pile surrounding soil are as follows: the soil density is $1800 \mathrm{~kg} / \mathrm{m}^{3}$; both the Poisson's ratio in the horizontal direction caused by the vertical stress and the Poisson's ratio in the orthogonal direction of the horizontal strain caused by the horizontal stress are 0.4 ; the damping coefficient is $1000 \mathrm{~N} \cdot \mathrm{m}^{-3} \cdot \mathrm{s}$; the shear modulus in the horizontal plane is $60 \mathrm{MPa}$; the shear modulus in the vertical plane is $G_{v 2}^{s}=$ $20 \mathrm{MPa}, 40 \mathrm{MPa}, 60 \mathrm{MPa}, 80 \mathrm{MPa}, 100 \mathrm{MPa}$, respectively.

Figure 2 shows the influence of the shear modulus of pile surrounding soil in the vertical plane on the dynamic response of pile. As shown in Figure 2(a), it can be noted that the velocity admittance curves oscillate about a mean amplitude as the frequency increases. As the shear modulus of pile surrounding soil in the vertical plane increases, the amplitude of resonance peaks gradually decreases, but the resonance frequency of velocity admittance curves almost remains unchanged. As shown in Figure 2(b), it is observed that the amplitude of the incident pulses and reflective wave signals decreases with the increase of the shear modulus of pile surrounding soil in the vertical plane. As the shear modulus of pile surrounding soil in the vertical plane increases, the raising phenomenon between the incident pulses and the primary reflective wave signals will be gradually aggravated, and the declining phenomenon between the primary reflective wave signals and the secondary reflective wave signals will also be gradually intensified.

After that, the influence of the shear modulus of pile surrounding soil in the horizontal plane on the dynamic response of pile is studied. Parameters of pile surrounding soil are as follows: the shear modulus in the vertical plane is $60 \mathrm{MPa}$, and the shear modulus in the horizontal plane is $G_{h 2}^{s}=20 \mathrm{MPa}, 40 \mathrm{MPa}, 60 \mathrm{MPa}, 80 \mathrm{MPa}, 100 \mathrm{MPa}$, respectively. The other parameters of soil-pile system are the same as those shown in the previous case.

Figure 3 shows the influence of the shear modulus of pile surrounding soil in the horizontal plane on the dynamic response of pile. As shown in Figure 3(a), it can be seen that the amplitude of resonance peaks gradually increases with the increase of the shear modulus of pile surrounding soil in the horizontal plane, but the change of resonance frequency can be ignored. As shown in Figure 3(b), it can be seen that the amplitude of the reflective wave signals decreases with the increase of the shear modulus of pile surrounding soil in the horizontal plane, but the decreasing ratio is small. The raising phenomenon between the primary reflective wave signals and the secondary reflective wave signals will be gradually intensified with the increase of the shear modulus of pile surrounding soil in the horizontal plane.

\subsection{Influence of the Anisotropy of Pile End Soil on the Dynamic} Response of Pile. In this section, the influence of the shear modulus of pile end soil in the vertical plane on the dynamic response of pile is firstly investigated. Parameters of pile surrounding soil are as follows: the soil density is $1800 \mathrm{~kg} / \mathrm{m}^{3}$; both the shear modulus in the horizontal plane and the shear modulus in the vertical plane are $60 \mathrm{MPa}$; both the Poisson's 


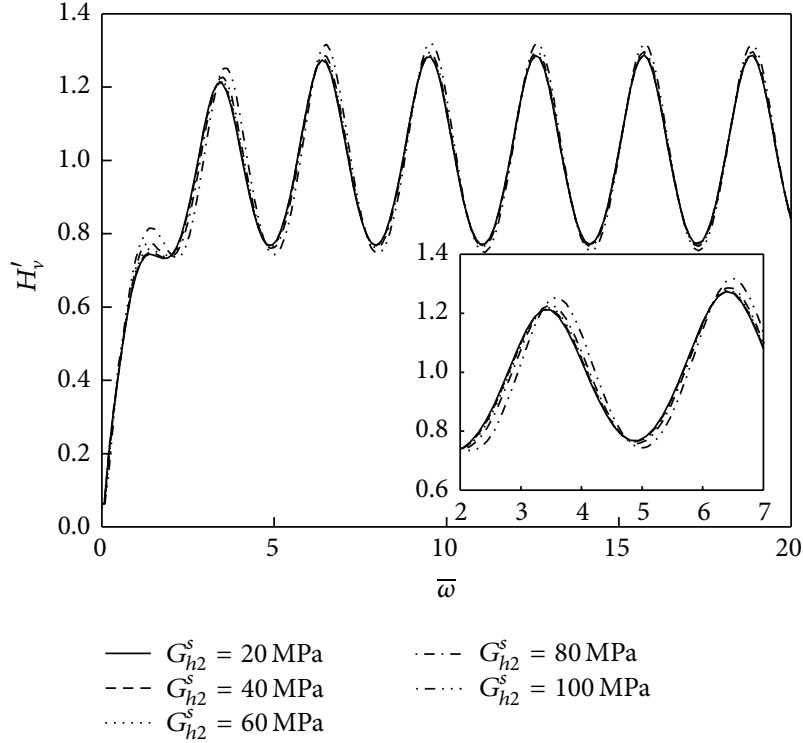

(a) Velocity admittance curves

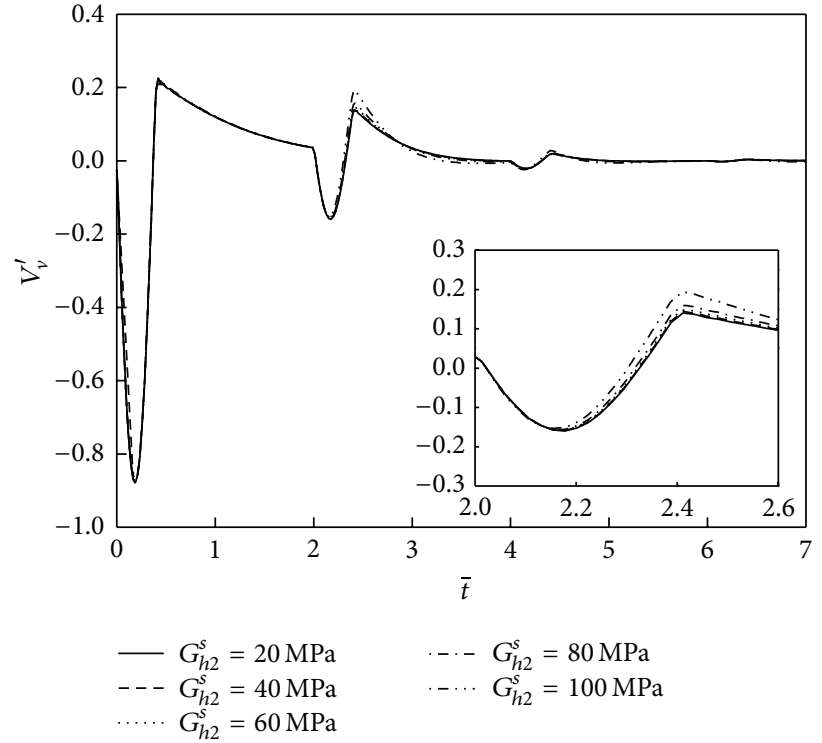

(b) Reflected wave signal curves

FIGURE 3: Influence of the shear modulus of pile surrounding soil in the horizontal plane on the dynamic response of pile.

ratio in the horizontal direction caused by the vertical stress and the Poisson's ratio in the orthogonal direction of the horizontal strain caused by the horizontal stress are 0.4; the damping coefficient is $1000 \mathrm{~N} \cdot \mathrm{m}^{-3} \cdot \mathrm{s}$. Parameters of pile end soil are as follows: the thickness is three times that of pile diameter; the soil density is $2000 \mathrm{~kg} / \mathrm{m}^{3}$; both the Poisson's ratio in the horizontal direction caused by the vertical stress and the Poisson's ratio in the orthogonal direction of the horizontal strain caused by the horizontal stress are 0.35 ; the damping coefficient is $1000 \mathrm{~N} \cdot \mathrm{m}^{-3} \cdot \mathrm{s}$; the shear modulus in the horizontal plane is $120 \mathrm{MPa}$; the shear modulus in the vertical plane is $G_{v 1}^{s}=80 \mathrm{MPa}, 100 \mathrm{MPa}, 120 \mathrm{MPa}, 140 \mathrm{MPa}$, $160 \mathrm{MPa}$, respectively.

Figure 4 shows the influence of the shear modulus of pile end soil in the vertical plane on the dynamic response of pile. As shown in Figure 4(a), as the shear modulus of pile end soil in the vertical plane increases, the amplitude of resonance peaks gradually decreases with the same resonance frequency, but the decreasing ratio is small. As shown in Figure 4(b), it can be seen that the amplitude of the reflective wave signals decreases with the increase of the shear modulus of pile surrounding soil in the vertical plane.

Then, the influence of the shear modulus of pile end soil in the horizontal plane on the dynamic response of pile is studied. Parameters of pile end soil are as follows: the shear modulus in the vertical plane is $120 \mathrm{MPa}$, and the shear modulus in the horizontal plane is $G_{h 1}^{s}=80 \mathrm{MPa}, 100 \mathrm{MPa}$, $120 \mathrm{MPa}, 140 \mathrm{MPa}, 160 \mathrm{MPa}$, respectively. The other parameters of soil-pile system are the same as those shown in the previous case.

Figure 5 shows the influence of the shear modulus of pile end soil in the horizontal plane on the dynamic response of pile. It can be seen that the influence of the shear modulus of pile end soil in the horizontal plane on the dynamic response of pile can be ignored.

\section{Conclusions}

By considering a pile embedded in layered transversely isotropic soil as a dynamic soil-pile interaction problem, the governing equations of soil-pile system are established when there is arbitrary vertical harmonic force acting on the pile head. Then, an analytical solution for the velocity response in the frequency domain and its corresponding semianalytical solution for the velocity response in the time domain have been derived by virtue of the transform technique and the separation of variables technique. An extensive parameter study has been undertaken to investigate the influence of the soil anisotropy on the vertical dynamic response of pile and the following conclusions have been obtained.

(1) Whether for the pile surrounding soil or for the pile end soil, it can be seen that the influence of the shear modulus in the vertical plane on the dynamic response of pile is more notable than the influence of the shear modulus in the horizontal plane on the dynamic response of pile. Therefore, the shear modulus of soil in the vertical plane plays a leading role in the dynamic response of pile when only the vertical wave effect of soil is taken into account.

(2) As the shear modulus of pile surrounding soil in the vertical plane increases, both the amplitude of the resonance peaks of velocity admittance curves and the reflective wave signals of reflected wave signal curves gradually decrease. As the shear modulus of pile surrounding soil in the horizontal plane increases, the 


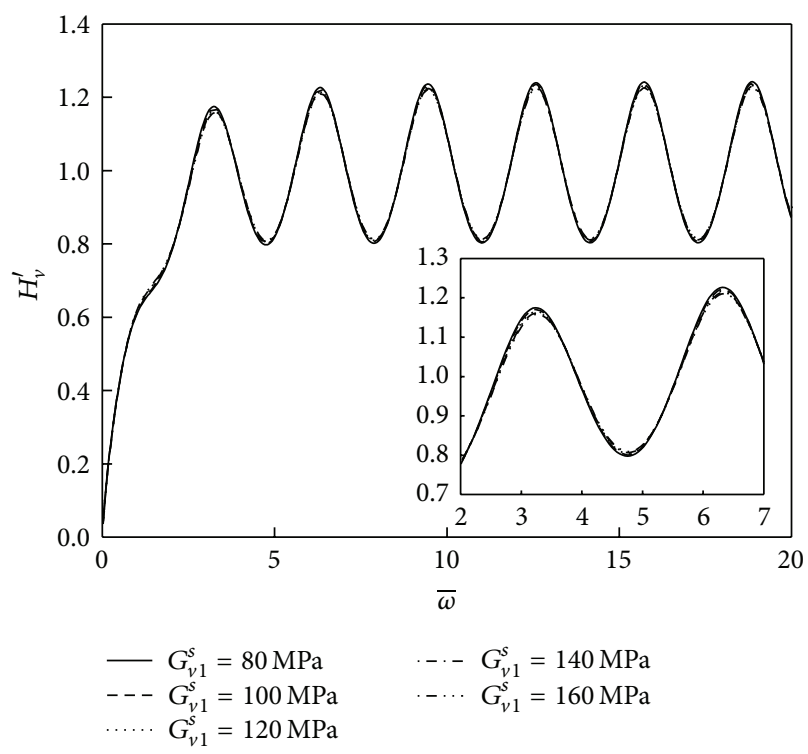

(a) Velocity admittance curves

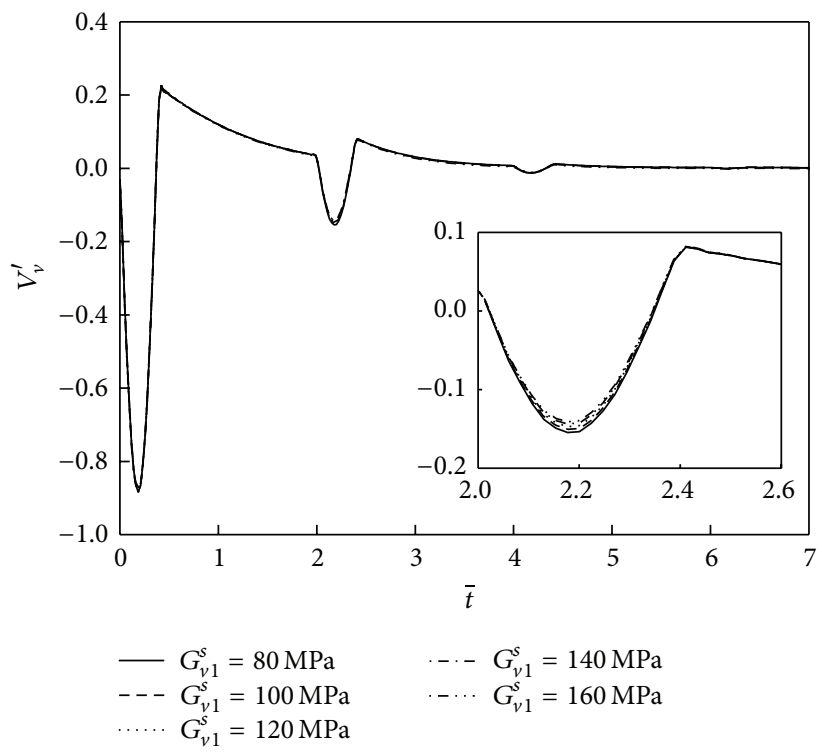

(b) Reflected wave signal curves

FIGURE 4: Influence of the shear modulus of pile end soil in the vertical plane on the dynamic response of pile.

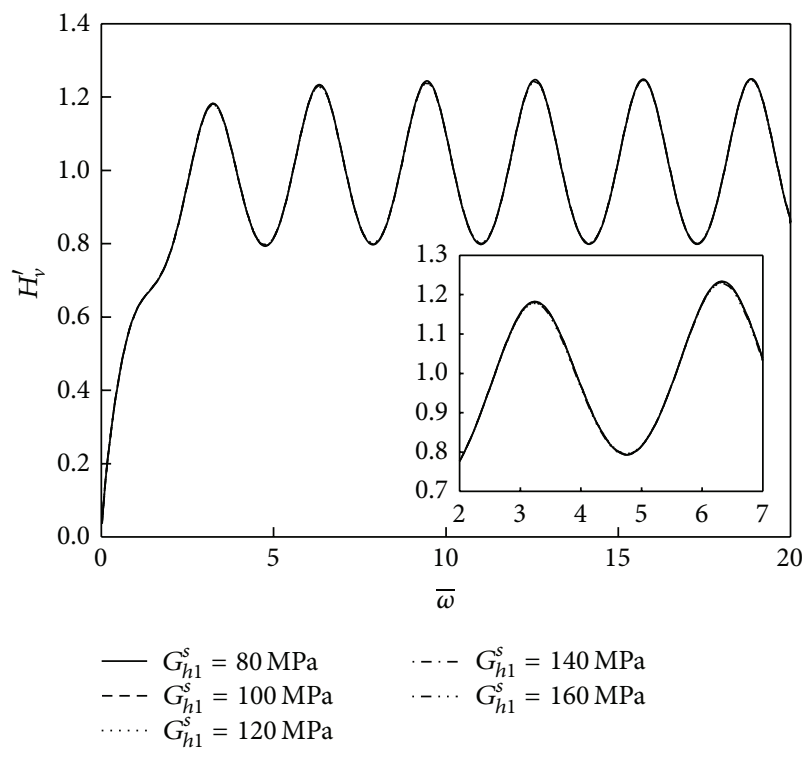

(a) Velocity admittance curves

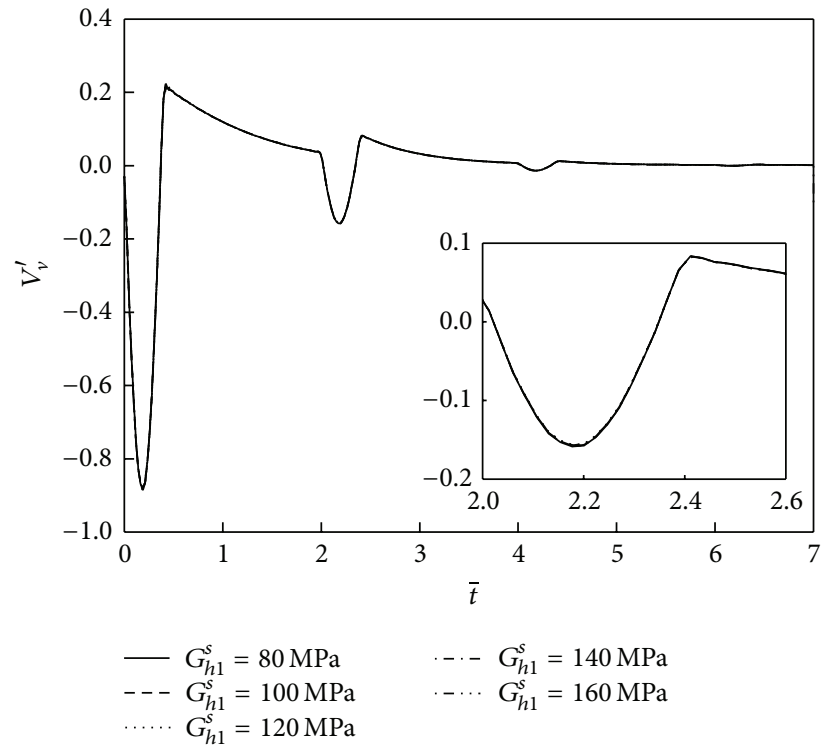

(b) Reflected wave signal curves

FIGURE 5: Influence of the shear modulus of pile end soil in the horizontal plane on the dynamic response of pile.

amplitude of the resonance peaks of velocity admittance curves gradually increases, but the reflective wave signals of reflected wave signal curves gradually decrease.

(3) As the shear modulus of pile end soil in the vertical plane increases, both the amplitude of the resonance peaks of velocity admittance curves and the reflective wave signals of reflected wave signal curves gradually decrease. The influence of the shear modulus of pile end soil in the horizontal plane on the dynamic response of pile can be ignored.

\section{Conflict of Interests}

The authors declare that there is no conflict of interests regarding the publication of this paper. 


\section{Acknowledgments}

This research is supported by the National Natural Science Foundation of China (Grant no. 51309207), the China Postdoctoral Science Foundation Funded Project (Grant nos. 2012M521495 and 2013T60759), and the Fundamental Research Funds for the Central Universities (Grant no. CUG120821).

\section{References}

[1] M. Novak and Y. O. Beredugo, "Vertical vibration of embedded footings," Journal of the Soil Mechanics and Foundations Division, vol. 98, no. 12, pp. 1291-1310, 1972.

[2] T. Nogami and K. Konagai, "Time domain axial response of dynamically loaded single piles," Journal of Engineering Mechanics, ASCE, vol. 112, no. 11, pp. 1241-1252, 1986.

[3] S. T. Liao and J. M. Roesset, "Dynamic response of intact piles to impulse loads," International Journal for Numerical and Analytical Methods in Geomechanics, vol. 21, no. 4, pp. 255-275, 1997.

[4] S. T. Liao and J. M. Roesset, "Identification of defects in piles through dynamic testing," International Journal for Numerical and Analytical Methods in Geomechanics, vol. 21, no. 4, pp. 277291, 1997.

[5] O. Michaelides, G. Gazetas, G. Bouckovalas, and E. Chrysikou, "Approximate non-linear dynamic axial response of piles," Geotechnique, vol. 48, no. 1, pp. 33-53, 1998.

[6] D. J. Liu, "Longitudinal waves in piles with exponentially varying cross sections," Chinese Journal of Geotechnical Engineering, vol. 30, no. 7, pp. 1066-1071, 2008.

[7] K. H. Wang, W. B. Wu, Z. Q. Zhang, and C. J. Leo, "Vertical dynamic response of an inhomogeneous viscoelastic pile," Computers and Geotechnics, vol. 37, no. 4, pp. 536-544, 2010.

[8] K. A. Kuo and H. E. M. Hunt, "An efficient model for the dynamic behaviour of a single pile in viscoelastic soil," Journal of Sound and Vibration, vol. 332, no. 10, pp. 2549-2561, 2013.

[9] M. Novak, "Dynamic stiffness and damping of piles," Canadian Geotechnical Journal, vol. 11, no. 4, pp. 574-598, 1974.

[10] M. Novak and F. Aboul-Ella, "Dynamic soil reaction for plane strain case," Journal of the Engineering Mechanical Division, vol. 104, no. 4, pp. 953-959, 1978.

[11] S. M. Mamoon and P. K. Banerjee, "Time-domain analysis of dynamically loaded single piles," Journal of Engineering Mechanics, vol. 118, no. 1, pp. 140-160, 1992.

[12] Y. C. Han, "Dynamic vertical response of piles in nonlinear soil," Journal of Geotechnical Engineering, vol. 123, no. 8, pp. 710-716, 1997.

[13] M. H. El Naggar and M. Novak, "Nonlinear lateral interaction in pile dynamics," Soil Dynamics and Earthquake Engineering, vol. 14, no. 2, pp. 141-157, 1995.

[14] M. H. El Naggar and M. Novak, "Nonlinear analysis for dynamic lateral pile response," Soil Dynamics and Earthquake Engineering, vol. 15, no. 4, pp. 233-244, 1996.

[15] G. Militano and R. K. N. D. Rajapakse, "Dynamic response of a pile in a multi-layered soil to transient torsional and axial loading," Geotechnique, vol. 49, no. 1, pp. 91-109, 1999.

[16] W. B. Wu, G. S. Jiang, B. Dou, and C. J. Leo, "Vertical dynamic impedance of tapered pile considering compacting effect," Mathematical Problems in Engineering, vol. 2013, Article ID 304856, p. 9, 2013.
[17] T. Nogami and M. Novak, "Soil-pile interaction in vertical vibration," Earthquake Engineering and Structural Dynamics, vol. 4, no. 3, pp. 277-293, 1976.

[18] R. K. N. D. Rajapakse, Y. Chen, and T. Senjuntichai, "Electroelastic field of a piezoelectric annular finite cylinder," International Journal of Solids and Structures, vol. 42, no. 11-12, pp. 3487-3508, 2005.

[19] T. Senjuntichai, S. Mani, and R. K. N. D. Rajapakse, "Vertical vibration of an embedded rigid foundation in a poroelastic soil," Soil Dynamics and Earthquake Engineering, vol. 26, no. 6-7, pp. 626-636, 2006.

[20] K. H. Wang, Z. Q. Zhang, C. J. Leo, and K. H. Xie, “Dynamic torsional response of an end bearing pile in saturated poroelastic medium," Computers and Geotechnics, vol. 35, no. 3, pp. 450-458, 2008.

[21] C. B. Hu and X. M. Huang, "A quasi-analytical solution to soil-pile interaction in longitudinal vibration in layered soils considering vertical wave effect on soils," Journal of Earthquake Engineering and Engineering Vibration, vol. 26, no. 4, pp. 205211, 2006.

[22] L. C. Liu, Q. F. Yan, and X. Yang, "Vertical vibration of single pile in soil described by fractional derivative viscoelastic model," Engineering Mechanics, vol. 28, no. 8, pp. 177-182, 2011.

[23] A. S. Veletsos and K. W. Dotson, "Vertical and torsional vibration of foundations in inhomogeneous media," Journal of Geotechnical Engineering, vol. 114, no. 9, pp. 1002-1021, 1988.

[24] K. W. Dotson and A. S. Veletsos, "Vertical and torsional impedances for radially inhomogeneous viscoelastic soil layers," Soil Dynamics and Earthquake Engineering, vol. 9, no. 3, pp. 110$119,1990$.

[25] M. H. El Naggar, "Vertical and torsional soil reactions for radially inhomogeneous soil layer," Structural Engineering and Mechanics, vol. 10, no. 4, pp. 299-312, 2000.

[26] H. D. Wang and S. P. Shang, "Research on vertical dynamic response of single-pile in radially inhomogeneous soil during the passage of Rayleigh waves," Journal of Vibration Engineering, vol. 19, no. 2, pp. 258-264, 2006.

[27] D. Y. Yang, K. H. Wang, Z. Q. Zhang, and C. J. Leo, "Vertical dynamic response of pile in a radially heterogeneous soil layer," International Journal for Numerical and Analytical Methods in Geomechanics, vol. 33, no. 8, pp. 1039-1054, 2009.

[28] S. G. Lekhnitskii, Theory of Anisotropic Elastic Bodies, Holdenday, San Francisco, Calif, USA, 1963.

[29] Y. M. Tsai, "Torsional vibrations of a circular disk on an infinite transversely isotropic medium," International Journal of Solids and Structures, vol. 25, no. 9, pp. 1069-1076, 1989.

[30] M. W. Liu and M. Novak, "Dynamic response of single piles embedded in transversely isotropic layered media," Earthquake Engineering \& Structural Dynamics, vol. 23, no. 11, pp. 1239-1257, 1994.

[31] R. Chen, C. F. Wan, S. T. Xue, and H. S. Tang, "Dynamic impedances of double piles in transversely isotropic layered media," Journal of Tongji University, vol. 31, no. 2, pp. 127-131, 2003.

[32] G. Chen, Y. Q. Cai, F. Y. Liu, and H. L. Sun, "Dynamic response of a pile in a transversely isotropic saturated soil to transient torsional loading," Computers and Geotechnics, vol. 35, no. 2, pp. 165-172, 2008.

[33] K. H. Wang, Z. Q. Zhang, C. J. Leo, and K. H. Xie, "Dynamic torsional response of an end bearing pile in transversely isotropic saturated soil," Journal of Sound and Vibration, vol. 327, no. 3-5, pp. 440-453, 2009. 
[34] W. B. Wu, K. H. Wang, D. Y. Yang, S. J. Ma, and B. N. $\mathrm{Ma}$, "Longitudinal dynamic response to the pile embedded in layered soil based on fictitious soil pile model," China Journal of Highway and Transport, vol. 25, no. 2, pp. 72-80, 2012.

[35] H. J. Ding, Transversely Isotropic Elastic Mechanics, Zhejiang University Publishing House, Hangzhou, China, 1997. 


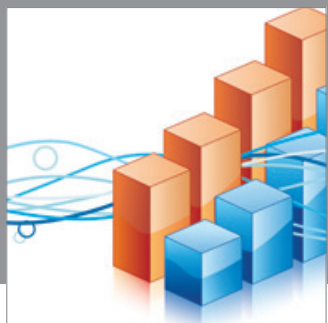

Advances in

Operations Research

mansans

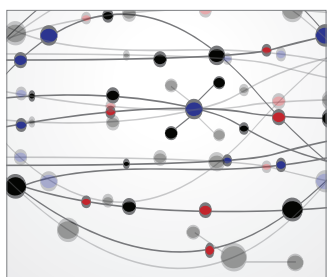

The Scientific World Journal
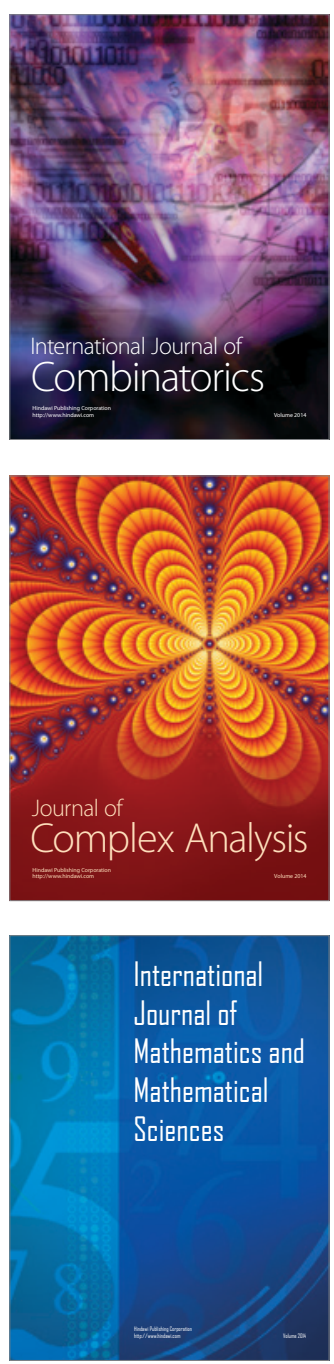
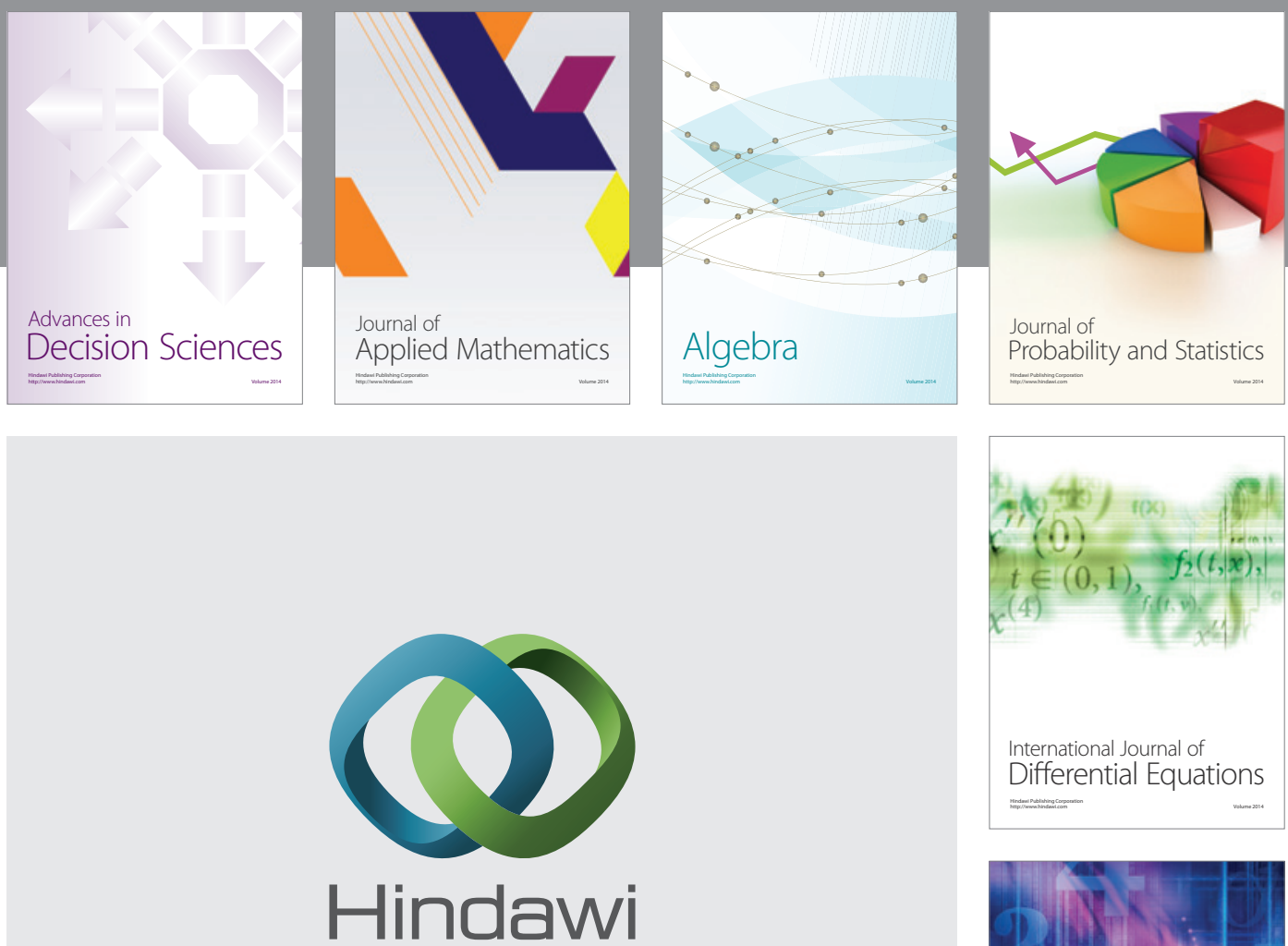

Submit your manuscripts at http://www.hindawi.com
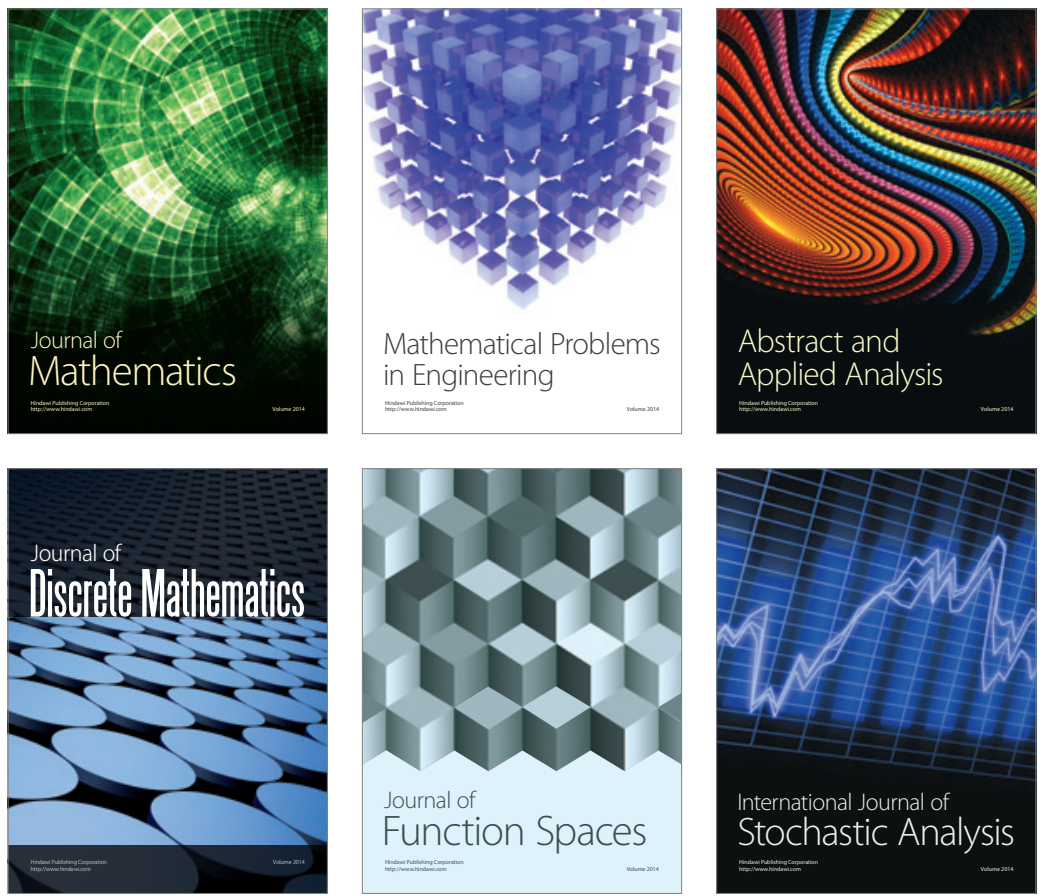

Journal of

Function Spaces

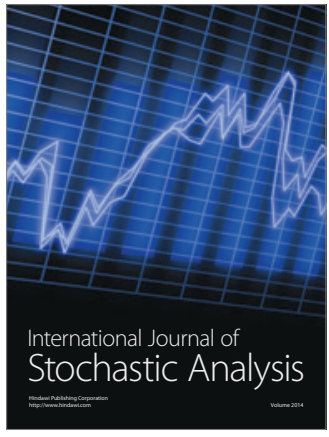

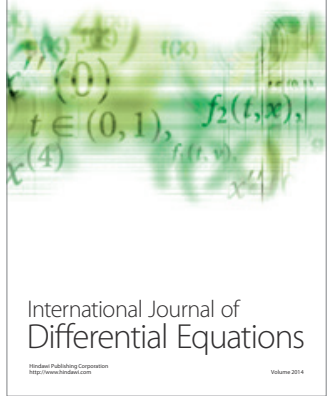
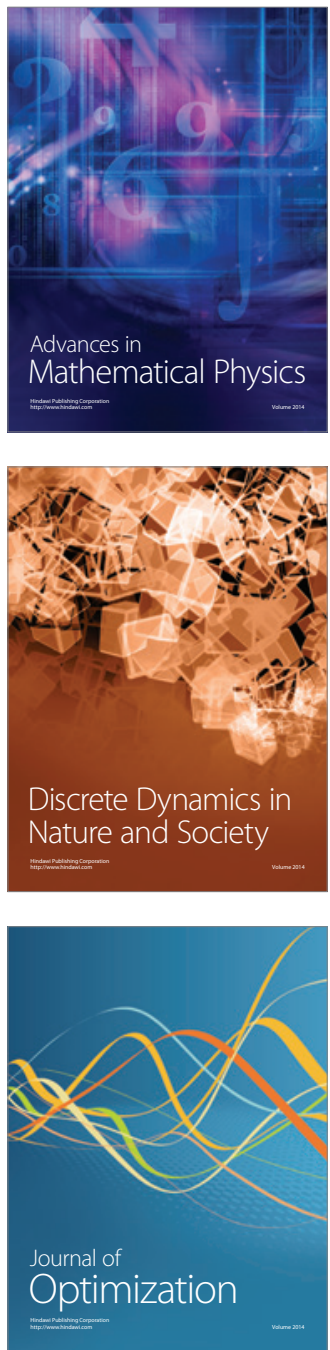